\title{
Sequential One-Pot Glycosylations Using 1-Hydroxyl and 1-Thiodonors
}

\author{
Jeroen D. C. Codée, Leendert J. van den Bos, Remy E. J. N. Litjens, Herman S. \\ Overkleeft, Jacques H. van Boom, Gijs A. van der Marel*
}

Leiden Institute of Chemistry, Leiden University, P. O. Box 9502, 2300 RA Leiden, The Netherlands.

General Procedures. All reactions were performed under argon atmosphere. Dichloromethane was refluxed with $\mathrm{P}_{2} \mathrm{O}_{5}$ and distilled before use. TTBP was synthesized as described by Crich et al. ${ }^{1}$ Traces of water in the donor and acceptor glycosides, diphenylsulfoxide and TTBP were removed by coevaporation with toluene and dichloroethane. Molecular sieves $(3 \AA)$ were flame dried before use. ${ }^{1} \mathrm{H}$ and ${ }^{13} \mathrm{C} \mathrm{NMR}$ spectra were recorded with a Bruker DPX 300 (300 and 75.1 MHz) or a Bruker AV 400 (400 and $100 \mathrm{MHz}$ ). ${ }^{1} \mathrm{H}$ NMR chemical shifts $(\delta)$ in $\mathrm{CDCl}_{3}$ are reported relative to tetramethylsilane. Mass spectra were recorded on a PE/SCIEX API 165 equipped with an Electrospray Interface (Perkin-Elmer). Optical rotations were recorded on a Propol automatic polarimeter in $\mathrm{CHCl}_{3}$.

\section{General procedure for the dehydrative condensations of 1-hydroxyl donors and thioglycosides:}

To a solution of the 1-hydroxyl donor (1.2-1.5 equiv to acceptor, $0.02 \mathrm{M}$ in DCM), $\mathrm{Ph}_{2} \mathrm{SO}$ (2.2 equiv to donor) and TTBP in $\mathrm{DCM}$ at $-60^{\circ} \mathrm{C}$ was added $\mathrm{Tf}_{2} \mathrm{O}$ (1.05 equiv to donor). The temperature was raised to $-40^{\circ} \mathrm{C}$ and stirred at this temperature for one hour. Then a solution of acceptor in DCM (0.1 M) was added and the reaction mixture was allowed to warm to room temperature. Dry $\mathrm{Et}_{3} \mathrm{~N}$ (10 equiv to donor) was added and the 
reaction mixture was washed with saturated $\mathrm{NaHCO}_{3}$ and water. After drying $\left(\mathrm{MgSO}_{4}\right)$ and concentration the residue was purified by column chromatography (ethyl acetate/petroleum ether or ethyl acetate/toluene).

\section{Ethyl 4- $O$-(2,3,4,6-tetra- $O$-benzyl- $\alpha / \beta$-D-glucopyranosyl)-2,3,6-tri- $O$-benzyl-1-thio- $\beta$ -} D-glucopyranoside (16): 2,3,4,6-tetra- $O$-benzyl- $\alpha / \beta$-D-glucopyranose 8 (97 mg, 0.18 mmol) was activated with $\mathrm{Ph}_{2} \mathrm{SO}(80 \mathrm{mg}, 0.40 \mathrm{mmol})$ and $\mathrm{Tf}_{2} \mathrm{O}(33 \mu \mathrm{L}, 0.20 \mathrm{mmol})$ in the presence of TTBP (112 mg. $0.45 \mathrm{mmol}$ ) as described above. Condensation with ethyl 2,3,6-tri- $O$-benzyl-1-thio- $\beta$-D-glucopyranoside 12 (74 mg, $0.15 \mathrm{mmol}$ ) gave disaccharide $16(88 \mathrm{mg}, \alpha / \beta 3: 1,87 \%)$ as an oil. $^{2}$

\section{3,4-di- $O$-benzoyl-6- $O$-tert-butyldimethylsilyl- $\alpha$-D-glucopyranose 1,2 -[phenyl phenyl} (3-O-benzyl-2,3-O-isopropylidene-1-thio- $\alpha$-D-mannopyranosid-6-yl) orthoacetate] (17): 2,3,4-tri- $O$-benzoyl-6- $O$-tert-butyldimethylsilyl- $\alpha$-D-glucopyranose 9 (124 mg, $0.20 \mathrm{mmol})$ was activated with $\mathrm{Ph}_{2} \mathrm{SO}(115 \mathrm{mg})$ and $\mathrm{Tf}_{2} \mathrm{O}(35 \mu \mathrm{L})$ in the presence of TTBP (152 mg) as described above. Condensation with phenyl 4-O-benzyl-2,3-Oisopropylidene-1-thio- $\alpha$-D-mannopyranoside $13(69 \mathrm{mg}, 0.17 \mathrm{mmol})$ gave after basic work-up (drying with $\mathrm{Na}_{2} \mathrm{SO}_{4}$ ) and purification using neutralized silicagel $\left(2 \% \mathrm{Et}_{3} \mathrm{~N}\right.$ added to the eluent) orthoester $17(136 \mathrm{mg}, 81 \%)$ as an oil. $\mathrm{R}_{f} 0.70(10 \%$ EtOAc in toluene). ${ }^{1} \mathrm{H}$ NMR (400 MHz, $\left.\mathrm{CDCl}_{3}\right): 8.08-7.13\left(\mathrm{~m}, 25 \mathrm{H}, \mathrm{CH}_{\text {arom }}\right.$ ), 5.81 (d, $1 \mathrm{H}, J=5.3$ Hz, H-1'), 5.73 (s, 1H, H-1), 5.62 (dd, 1H, $J=1.5 \mathrm{~Hz}, J=2.3 \mathrm{~Hz}, \mathrm{H}-3$ '), 5.35 (d, 1H, $J=$ $8.6 \mathrm{~Hz}, \mathrm{H}-4$ '), 4.81 (d, 1H, J = 11.5 Hz, CHH Bn), 4.47 (m, 2H, H-2', CHH Bn), 4.31 (m, 2H, H-2, H-3), 4.24 (m, 1H, H-5), 3.82 (m, 1H, H-5'), 3.72 (m, 2H, H-6', H-6'), 3.56 (dd, $1 \mathrm{H}, J=2.0 \mathrm{~Hz}, J=10.6 \mathrm{~Hz}, \mathrm{H}-6), 3.43$ (m, 2H, H-4, H-6), 1.47 (s, 3H, $\mathrm{CH}_{3}$ isoprop), 1.34 (s, $3 \mathrm{H}, \mathrm{CH}_{3}$ isoprop), 0.79 (s, 9H, $\mathrm{CH}_{3} t \mathrm{Bu}$ TBS), -0.05 (s, $3 \mathrm{H}, \mathrm{CH}_{3} \mathrm{TBS}$ ), -0.06 (s,

$\left.3 \mathrm{H}, \mathrm{CH}_{3} \mathrm{TBS}\right) ;{ }^{13} \mathrm{C} \mathrm{NMR}\left(100 \mathrm{MHz}, \mathrm{CDCl}_{3}\right): \delta=165.0,164.4,137.9,135.2,133.4$, 133.2, 132.0, 130.0, 129.8, 129.5, 129.4, 129.3, 128.8, 128.4, 128.3, 128.2, 127.7, 127.55, 127.48, 126.6, 120.9, 109.5, 97.6, 83.9, 78.3, 76.3, 76.2, 72.7, 71.9, 69.5, 69.2, 69.1, 68.2, 63.7, 63.1, 27.9, 26.3, 25.7, 18.1, -5.5; ES-MS: $m / z 1013.6[\mathrm{M}+\mathrm{Na}]^{+}$. 


\section{3,4,6-tri- $O$-pivaloyl- $\alpha$-D-glucopyranose 1,2 -[tert-butyl phenyl (3-O-benzyl-2,3-O}

isopropylidene-1-thio- $\alpha$-D-mannopyranosid-6-yl) orthoacetate] (18): 2,3,4,6-tetra- $O$ pivaloyl- $\alpha$-D-glucopyranose 10 (77 $\mathrm{mg}, 0.15 \mathrm{mmol}$ ) was condensed with phenyl 3-Obenzyl-2,3-O-isopropylidene-1-thio- $\alpha$-D-mannopyranoside (13) as described for the condensation of $\mathbf{9}$ and $\mathbf{1 3}$ to give orthoester $\mathbf{1 8}(85 \%) . \mathrm{R}_{f} 0.85\left(25 \%\right.$ EtOAc in PE). $[\alpha]^{25}$ $+41.0\left(c=0.50, \mathrm{CHCl}_{3}\right) ;{ }^{1} \mathrm{H} \mathrm{NMR}\left(400 \mathrm{MHz}, \mathrm{CDCl}_{3}\right): \delta=7.47-7.27\left(\mathrm{~m}, 10 \mathrm{H}, \mathrm{CH}_{\text {arom }}\right)$, 5.66 (s, 1H, H-1), 5.51 (d, 1H, J=5.6 Hz, H-1'), 5.17 (dd, 1H, J=3.3 Hz, J=4.2 Hz, H3'), 4.89 (m, 2H, H-4', CHH Bn), 4.62 (d, 1H, J = 11.2 Hz, CHH Bn), 4.30 (m, 2 H, H-2, H-3), 4.29 (dd, 1H, J = 3.2 Hz, $J=5.6$ Hz, H-2'), 4.14 (m, 4H, H-5, H-5', H-6', H-6'), 3.75 (m, 3H, H-4, H-6, H-6), 1.50 (s, 3H, $\mathrm{CH}_{3}$ isoprop), 1.38 (s, 3H, $\mathrm{CH}_{3}$ isoprop), 1.24 (s, 9H, $\mathrm{CH}_{3}$ Piv), 1.21 (s, 18H, 2 x CH 3 Piv), 1.03 (s, 9H, $\mathrm{CH}_{3} \mathrm{Piv}$ ); ${ }^{13} \mathrm{C}$ NMR (100 MHz, $\left.\mathrm{CDCl}_{3}\right): \delta=177.5,176.8,176.3,138.1,133.1,131.8,129.1,128.3,128.0,127.9,127.7$, 124.6, 109.6, 96.9, 84.0, 78.4, 76.3, 75.6, 74.3, 73.3, 70.8, 69.9, 67.8, 67.3, 63.4, 62.7, 39.4, 38.8, 38.64, 38.55, 28.0, 27.1, 27.0, 26.5, 25.7; ES-MS: $m / z 923.5[\mathrm{M}+\mathrm{Na}]^{+}$.

\section{Phenyl 6-O-(2,3,4,6-tetra- $O$-pivaloyl- $\alpha$-D-glucopyranosyl)-3- $O$-benzyl-2,3-O-} isopropylidene-1-thio- $\alpha$-D-mannopyranoside (19): 2,3,4,6-tetra- $O$-pivaloyl- $\alpha$-Dglucopyranose 10 (77 mg, $0.15 \mathrm{mmol})$ was activated with $\mathrm{Ph}_{2} \mathrm{SO}(66 \mathrm{mg})$ and $\mathrm{Tf}_{2} \mathrm{O}(28$ $\mu \mathrm{L}$ ) in the presence of TTBP (34 mg, 1.0 equiv to donor) and condensed with phenyl 3$O$-benzyl-2,3-O-isopropylidene-1-thio- $\alpha$-D-mannopyranoside (13) to give disaccharide 19 in $80 \%$ yield. $\mathrm{R}_{f} 0.80\left(25 \%\right.$ EtOAc in PE). $[\alpha]^{25}+56.4\left(c=0.83, \mathrm{CHCl}_{3}\right) ;{ }^{1} \mathrm{H} \mathrm{NMR}$ (300 MHz, $\left.\mathrm{CDCl}_{3}\right)$ : 7.53-7.26 (m, $\left.10 \mathrm{H}, \mathrm{CH}_{\text {arom }}\right), 5.76(\mathrm{~s}, 1 \mathrm{H}, \mathrm{H}-1), 5.12(\mathrm{t}, 1 \mathrm{H}, J=9.4$ Hz, H-3'), 4.94 (m, 2H, H-2', H-4'), 4.88 (d, 1H, $J=11.6$ Hz, CHH Bn), 4.58 (d, 1H, J= $11.6 \mathrm{~Hz}, \mathrm{CH} H \mathrm{Bn}), 4.43$ (d, 1H, J = 8.0 Hz, H-1'), 4.34 (m, 2H, H-2, H-3), 4.20 (m, 1H, H-5), 4.13 (dd, 1H, J = 1.6 Hz, J = 12.2 Hz, H-6'), 3.96 (m, 2H, H-6, H-6'), 3.83 (dd, 1H, $J=2.5 \mathrm{~Hz}, J=11.9 \mathrm{~Hz}, \mathrm{H}-4), 3.40$ (m, 1H, H-5'), 1.49 (s, 3H, $\mathrm{CH}_{3}$ isoprop), 1.37 (s, 3H, $\mathrm{CH}_{3}$ isoprop), 1.18 (s, 9H, $\mathrm{CH}_{3} \mathrm{Piv}$ ), 1.16 (s, 9H, $\mathrm{CH}_{3} \mathrm{Piv}$ ), 1.13 (s, 9H, $\mathrm{CH}_{3} \mathrm{Piv}$ ), 1.10 (s, 9H, $\mathrm{CH}_{3}$ Piv); ${ }^{13} \mathrm{C}$ NMR (100 MHz, $\left.\mathrm{CDCl}_{3}\right): \delta=178.9,178.0,177.2,138.8,134.4$, $131.9,130.2$, 129.2, 128.7, 128.6, 128.5, 110.4, 101.0, 84.5, 79.0, 77.1, 76.8, 73.5, 73.1, 
72.9, 71.9, 71.8, 68.83, 68.80, 62.8, 39.6, 39.5, 28.7, 28.0, 27.92, 27.87, 27.1; ES-MS: $\mathrm{m} / z 923.6[\mathrm{M}+\mathrm{Na}]^{+}$.

Ethyl 6-O-(2,3,5,6-tetra- $O$-benzoyl- $\alpha$-D-galactofuranosyl)-2,3,4-tri- $O$-benzyl-1-thio$\beta$-D-glucopyranoside (20): Condensation of $\mathbf{1 1}$ and $\mathbf{1 4}$ was executed as described for the condensation of $\mathbf{8}$ and $\mathbf{1 2}$ to give disaccharide $\mathbf{2 0}$ (68\% yield). $\mathrm{R}_{f} 0.85$ (40\% EtOAc in PE). $[\alpha]^{25}{ }_{\mathrm{D}}+3.8\left(c=1.0, \mathrm{CHCl}_{3}\right) ;{ }^{1} \mathrm{H}$ NMR $\left(400 \mathrm{MHz}, \mathrm{CDCl}_{3}\right): 8.08-7.22(\mathrm{~m}, 35 \mathrm{H}$, $\mathrm{CH}_{\text {arom }}$ ), 6.17 (m, 1H, H-5'), 5.61 (d, 1H, $\left.J=4.9 \mathrm{~Hz}, \mathrm{H}-3^{\prime}\right), 5.47$ (d, 1H, $J=0.7 \mathrm{~Hz}, \mathrm{H}-2^{\prime}$ ), 5.35 (s, 1H, H-1'), 4.92 (d, 1H, $J=10.7 \mathrm{~Hz}, \mathrm{CHH} \mathrm{Bn}), 4.89$ (d, 1H, $J=10.5 \mathrm{~Hz}, \mathrm{CHH}$ Bn), 4.86 (d, 1H, $J=10.8$ Hz, CHH Bn), 4.79 (m, 4H, H-4', H-6', H-6', CHH Bn), 4.58 $(\mathrm{d}, 1 \mathrm{H}, J=10.2 \mathrm{~Hz}, \mathrm{CH} H \mathrm{Bn}), 4.46(\mathrm{~d}, 1 \mathrm{H}, J=9.8 \mathrm{~Hz}, \mathrm{CH} H \mathrm{Bn}), 4.04$ (dd, $1 \mathrm{H}, J=1.5$ $\mathrm{Hz}, J=11.1 \mathrm{~Hz}, \mathrm{H}-6), 3.79$ (dd, 1H, $J=4.6 \mathrm{~Hz}, J=11.1 \mathrm{~Hz}$ ), 3.67 (m, 2H, H-3, H-4), 3.48 (m, 1H, H-5), 3.22 (m, 1H, H-2), 2.67 (m, 2H, $\mathrm{CH}_{2} \mathrm{SEt}$ ), 1.21 (t, $3 \mathrm{H}, J=7.4 \mathrm{~Hz}$, $\left.\mathrm{CH}_{3} \mathrm{SEt}\right) ;{ }^{13} \mathrm{C}$ NMR $\left(100 \mathrm{MHz}, \mathrm{CDCl}_{3}\right): \delta=166.1,165.8,165.5,165.2,138.5,137.93$, 137.88, 133.4, 133.2, 133.1, 132.9, 129.71, 129.67, 129.5, 129.0, 128.8, 128.4, 128.2, $128.0,127.6,105.2,86.4,84.8,81.8,81.7,81.6,78.3,77.6,75.7,75.4,75.0,70.4,65.3$, 64.0, 24.9, 15.0; ES-MS: $m / z$ 1095.3 [M + Na $]^{+}$.

\section{Phenyl}

3-O-(2,3,5,6-tetra- $O$-benzoyl- $\alpha$-D-galctofuranosyl)-2-azido-4,6- $O$ -

benzylidene-2-deoxy-1-seleno- $\alpha$-D-galactopyranoside (21): Condensation of 11 and 15 was executed as described for the condensation of $\mathbf{8}$ and $\mathbf{1 2}$ to give disaccharide $\mathbf{2 1}$ (56\% yield). $\mathrm{R}_{f} 0.55$ (20\% EtOAc in PE). $[\alpha]^{25}{ }_{\mathrm{D}}+83.6\left(c=1.0, \mathrm{CHCl}_{3}\right) ;{ }^{1} \mathrm{H} \mathrm{NMR}(400 \mathrm{MHz}$, $\mathrm{CDCl}_{3}$ ): 8.01-7.03 (m, 30H, $\left.\mathrm{CH}_{\text {arom }}\right), 6.08(\mathrm{~m}, 1 \mathrm{H}, \mathrm{H}-5$ '), 6.07 (d, $1 \mathrm{H}, J=5.2 \mathrm{~Hz}, \mathrm{H}-1)$, 5.69 (s, 1H, CH Ph), 5.67 (d, 1H, J = 5.0 Hz, H-3'), 5.58 (d, 1H, J =0.9 Hz, H-2'), 5.53 (s, 1H, H-1'), 4.72 (m, 3H, H-4', H-6', H-6'), 4.50 (m, 1H, H-4), 4.47 (dd, 1H, $J=5.2$ Hz, $J=$ 10.4, H-2), 4.04 (m, 3H, H-3, H-5, H-6), 3.91 (d, $J=11.1 \mathrm{~Hz}, \mathrm{H}-6) ;{ }^{13} \mathrm{C}$ NMR $(100 \mathrm{MHz}$, $\left.\mathrm{CDCl}_{3}\right): \delta=166.2,165.6,165.2,137.3,133.9,133.4,133.3,133.2,129.9,129.8,129.7$, 129.4, 129.3, 129.1 129.0, 128.81, 128.75, 128.2, 128.0, 127.7, 125.8, 107.5, 100.6, 85.0, $82.5,81.9,78.4,77.3,75.2,70.2,68.9,64.9,63.4,59.7$. ES-MS: $m / z 1034.5[\mathrm{M}+\mathrm{Na}]^{+}$. 


\section{Phenyl 3-O-(2,3,4,6-tetra- $O$-benzyl- $\alpha$-D-galactopyranosyl)-4- $O$-acetyl-2,6-di- $O$ -}

benzoyl-1-thio- $\beta$-D-galactopyranoside (24): Activation of 22 was executed as described for the activation of $\mathbf{8}$. Acceptor $\mathbf{2 3}$ was added and the reaction mixture was kept at $40^{\circ} \mathrm{C}$ for 1 hour, after which it was slowly warmed to $0^{\circ} \mathrm{C}$. Standard work-up and purification gave disaccharide 24 in $64 \%$. $\mathrm{R}_{f} 0.80$ (33\% EtOAc in PE). $[\alpha]^{25}+90.8(c=$ 1.0, $\left.\mathrm{CHCl}_{3}\right) ;{ }^{1} \mathrm{H} \mathrm{NMR}\left(400 \mathrm{MHz}, \mathrm{CDCl}_{3}\right): \delta=8.12-7.14\left(\mathrm{~m}, 35 \mathrm{H}, \mathrm{CH}_{\text {arom }}\right), 5.67(\mathrm{~d}, 1 \mathrm{H}$, $J=2.8 \mathrm{~Hz}, \mathrm{H}-4), 5.61(\mathrm{t}, 1 \mathrm{H}, J=9.9 \mathrm{~Hz}, \mathrm{H}-2), 5.22(\mathrm{~d}, 1 \mathrm{H}, J=3.2 \mathrm{~Hz}, \mathrm{H}-1$ '), 4.81 (d, $1 \mathrm{H}, J=10.1 \mathrm{~Hz}, \mathrm{H}-1), 4.76$ (d, 1H, $J=11.4 \mathrm{~Hz}, \mathrm{CHH} \mathrm{Bn}), 4.65$ (s, 2H, $\mathrm{CH}_{2} \mathrm{Bn}$ ), 4.64 (d, 1H, $J=12.2 \mathrm{~Hz}, \mathrm{CHH} \mathrm{Bn),} 4.48$ (m, 2H, H-6, CHH Bn), 4.36 (m, 4 H, H-6, 3xCHH Bn), 4.16 (dd, 1H, $J=3.0 \mathrm{~Hz}, J=9.7 \mathrm{~Hz}, \mathrm{H}-3$ ), 3.93 (m, 3H, H-2', H-5, H-5'), 3.75 (dd, 1H, $J$ $\left.=2.6 \mathrm{~Hz}, J=10.1 \mathrm{~Hz}, \mathrm{H}-3^{\prime}\right), 3.44$ (dd, 1H, $\left.J=7.3 \mathrm{~Hz}, J=9.6 \mathrm{~Hz}, \mathrm{H}-6^{\prime}\right), 3.23$ (bs, $1 \mathrm{H}, \mathrm{H}-$ 4'), $3.20(\mathrm{dd}, 1 \mathrm{H}, J=5.2 \mathrm{~Hz}, J=9.6 \mathrm{~Hz}, \mathrm{H}-6$ ) $), 1.89$ (s, 3H, $\mathrm{CH}_{3} \mathrm{Ac}$ ); ${ }^{13} \mathrm{C}$ NMR (100 $\left.\mathrm{MHz}, \mathrm{CDCl}_{3}\right): \delta=170.3,165.9,164.8,138.6,138.5,138.3,138.2,133.4,133.2,131.8$, 129.8, 129.7, 129.5, 129.3, 128.7, 128.4, 127.8, 127.6, 127.5, 127.4, 93.3, 87.0, 78.7, 75.5, 74.8, 74.7, 74.3, 73.21, 73.15, 73.0, 72.1, 69.9, 69.4, 68.9, 65.1, 62.7, 20.4; ESMS: $m / z 1067.5[\mathrm{M}+\mathrm{Na}]^{+}, 1083.4[\mathrm{M}+\mathrm{K}]^{+}$.

\section{3-azido-1-O-\{4-O-[3-O-(2,3,4,6-tetra- $O$-benzyl- $\alpha$-D-galactopyranosyl)-4- $O$-acetyl-2,6- di- $O$-benzoyl- $\beta$-D-galactopyranosyl]-3,6-di- $O$-benzyl-2-deoxy-2-phtalimido- $\beta$-D- glucopyranosyl\}propanol (26):}

From 24 and 25: Disaccharide 24 (0.02 $\mathrm{M}$ in DCM) was activated with $\mathrm{Ph}_{2} \mathrm{SO}$ (2.0 equiv to donor) and $\mathrm{Tf}_{2} \mathrm{O}$ (1.1 equiv to donor) in the presence of TTBP (2.5 equiv to donor) for 10 min at $-60^{\circ} \mathrm{C}$. Acceptor 25 (1.5 equiv to donor) was added and the reaction mixture was slowly warmed to $0^{\circ} \mathrm{C}$. Standard work-up and purification gave trisaccharide 26 (69\% yield).

One-pot procedure: 2,3,4,6-Tetra-O-benzyl- $\alpha$-D-galactopyranose 22 (68 mg, 0.125 $\mathrm{mmol}), \mathrm{Ph}_{2} \mathrm{SO}$ (51 mg, $\left.0.250 \mathrm{mmol}\right)$, and TTBP $(93 \mathrm{mg}, 0.375 \mathrm{mmol}$ ) were dissolved in DCM $(5 \mathrm{~mL})$ and the reaction mixture was cooled to $\left.-60^{\circ} \mathrm{C} . \mathrm{Tf}_{2} \mathrm{O}(22 \mu \mathrm{L}, 0.13 \mathrm{mmol})\right)$ was added and the reaction mixture was brought to $-40^{\circ} \mathrm{C}$. Stirring was continued for 1 
hour at this temparature, after which phenyl 4- $O$-acetyl-2,6-di- $O$-benzoyl-1-thio- $\beta$-Dgalactopyranoside 23 (52 $\mathrm{mg}, 0.100 \mathrm{mmol}$ in $1 \mathrm{~mL}$ DCM) was added. The reaction mixture was kept at $-40^{\circ} \mathrm{C}$ for 1 hour after which it was slowly warmed to $0^{\circ} \mathrm{C}$. After 30 min at $0^{\circ} \mathrm{C}$ the reaction mixture was cooled to $-60^{\circ} \mathrm{C}$ and a $\mathrm{Tf}_{2} \mathrm{O}(20 \mu \mathrm{L}, 0.12 \mathrm{mmol})$ was added. After the reaction was kept at $-60^{\circ} \mathrm{C}$ for $10 \mathrm{~min}, 3$-azido-1-O-(3,6-di-O-benzyl-2deoxy-2-phtalimido- $\beta$-D-glucopyranosyl)propanol 25 (86 mg, $0.15 \mathrm{mmol}$ ) was added. The reaction was slowly warmed to $0^{\circ} \mathrm{C}$ after which it was quenched with $\mathrm{Et}_{3} \mathrm{~N}(0.2 \mathrm{~mL})$. Standard work-up and purification gave the $\alpha$-Gal epitope $26(121 \mathrm{mg}, 0.082 \mathrm{mmol}$, $80 \%) . \mathrm{R}_{f} 0.60$ (33\% EtOAc in PE). $[\alpha]_{\mathrm{D}}^{25}+39.2\left(c=0.75, \mathrm{CHCl}_{3}\right) ;{ }^{1} \mathrm{H}$ NMR $(400 \mathrm{MHz}$, $\left.\mathrm{CDCl}_{3}\right): \delta=8.10-6.83$ (m, 44H, $\mathrm{H}_{\text {arom }}$ ), 5.54 (m, 2H, H-2', H-4'), 5.14 (d, 1H, $J=3.3 \mathrm{~Hz}$, H-1"), 5.00 (d, 1H, $J=8.5 \mathrm{~Hz}, \mathrm{H}-1), 4.91$ (d, 1H, $J=12.4 \mathrm{~Hz}, \mathrm{C} H \mathrm{H} \mathrm{Bn}), 4.78$ (d, 1H, $J=$ $\left.8.1 \mathrm{~Hz}, \mathrm{H}-1^{\prime}\right), 4.74$ (d, 1H, $\left.J=11.4 \mathrm{~Hz}, \mathrm{CHH} \mathrm{Bn}\right), 4.63$ (s, 2H, $\left.\mathrm{CH}_{2} \mathrm{Bn}\right), 4.62$ (d, 1H, $J=$ $11.8 \mathrm{~Hz}, \mathrm{CHH} \mathrm{Bn}), 4.55(\mathrm{~d}, 1 \mathrm{H}, J=12.0 \mathrm{~Hz}, \mathrm{C} H \mathrm{H} \mathrm{Bn}), 4.51(\mathrm{~d}, 1 \mathrm{H}, J=12.4 \mathrm{~Hz}, \mathrm{CH} H$ Bn), 4.44 (d, 1H, $J=11.8 \mathrm{~Hz}, \mathrm{CHH} \mathrm{Bn}), 4.42(\mathrm{~d}, 1 \mathrm{H}, J=11.8 \mathrm{~Hz}, \mathrm{CH} H \mathrm{Bn}), 4.35$ (d, 1H, $J=12.0 \mathrm{~Hz}, \mathrm{CH} H \mathrm{Bn}$ ), 4.29 (m, 3H, CHH Bn, CHH BN, H-3), 4.22 (dd, 1H, $J=6.5 \mathrm{~Hz}$, $\left.J=11.3 \mathrm{~Hz}, \mathrm{H}-6^{\prime}\right), 4.13$ (m, 2H, H-6', H-2), 4.05 (dd, 1H, $\left.J=8.5 \mathrm{~Hz}, J=9.9 \mathrm{~Hz}, \mathrm{H}-4\right)$, 3.99 (dd, 1H, $\left.J=3.4 \mathrm{~Hz}, J=10.2 \mathrm{~Hz}, \mathrm{H}-3^{\prime}\right), 3.91$ (dd, 1H, $\left.J=3.3 \mathrm{~Hz}, J=10.2 \mathrm{~Hz}, \mathrm{H}-2 "\right)$, 3.85 (bt, $1 \mathrm{H}, J=6.9 \mathrm{~Hz}, \mathrm{H}-5$ "), 3.75 (m, 1H, O- $\underline{\mathrm{CH}}_{2}-\mathrm{CH}_{2}$ ), 3.67 (m, 2H, H-5', H-6), 3.58 (m, 2H, H-6, H-3"), 3.41 (m, 1H, H-5), 3.38 (m, 2H, H-6", O-C $\underline{H}_{2}-\mathrm{CH}_{2}$ ), 3.25 (dd, 1H, $J$ $=1.2 \mathrm{~Hz}, J=2.6 \mathrm{~Hz}, \mathrm{H}-4 "), 3.21$ (dd, $1 \mathrm{H}, J=5.9 \mathrm{~Hz}, J=9.4 \mathrm{~Hz}, \mathrm{H}-6 "), 3.08$ (m, 2H, $\mathrm{CH}_{2} \mathrm{~N}_{3}$ ), 1.81 (s, $3 \mathrm{H}, \mathrm{CH}_{3} \mathrm{Ac}$ ), 1.64 (m, $2 \mathrm{H}, \mathrm{CH}_{2} \mathrm{CH}_{2} \mathrm{CH}_{2}$ ); ${ }^{13} \mathrm{C} \mathrm{NMR}\left(100 \mathrm{MHz}, \mathrm{CDCl}_{3}\right.$ ): $\delta=170.2,166.0,164.6,138.7,138.4,138.2,138.1,133.7,133.3,133.2,131.5,129.8$, 129.7, 129.3, 128.5, 128.4, 128.3, 128.1, 127.9, 127.8, 127.7, 127.6, 127.5, 127.4, 127.3, $126.9,123.2,100.8,98.3,94.1,78.8,78.3,76.9,75.5,74.8,74.7,74.5,73.5,73.3,73.2$, 73.1, 72.3, 71.5, 71.0, 69.8, 69.2, 67.8, 65.9, 65.0, 61.7, 55.7, 48.0, 28.8, 20.4; ES-MS: $\mathrm{m} / \mathrm{z} 1529.8[\mathrm{M}+\mathrm{Na}]^{+}$.

\section{Ethyl 3-O-[methyl $\quad(2,3,4-$ tri- $O$-benzoyl- $\alpha$-D-glucopyranosyluronate)]-4,6-O-} benzylidene-2-deoxy-2-phtalimido-1-thio- $\beta$-D-glucopyranoside (29): Methyl (2,3,4tri-O-benzoyl- $\alpha$-D-glucopyranosyluronate) 27 was activated with $\mathrm{Ph}_{2} \mathrm{SO}$ and $\mathrm{Tf}_{2} \mathrm{O}$ in the 
presence of TTBP (1.0 equiv to donor) as described above. Condensation with ethyl 4,6$O$-benzylidene-2-deoxy-2-phtalimido-1-thio- $\beta$-D-glucopyranoside 28 gave after work-up and purification disaccharide 29 in $58 \%$ yield. $\mathrm{R}_{f} 0.60$ (20\% EtOAc in toluene). $[\alpha]^{25}$ $+14.4\left(c=0.50, \mathrm{CHCl}_{3}\right) ;{ }^{1} \mathrm{H}$ NMR $\left(400 \mathrm{MHz}, \mathrm{CDCl}_{3}\right): \delta=7.80-7.15\left(\mathrm{~m}, 24 \mathrm{H}, \mathrm{CH}_{\text {arom }}\right)$, 5.64 (t, $\left.1 \mathrm{H}, J=9.4 \mathrm{~Hz}, \mathrm{H}-3^{\prime}\right), 5.57$ (s, 1H, CH Ph), 5.44 (t, 1H, $J=9.4 \mathrm{~Hz}, \mathrm{H}-4$ '), 5.32 $\left(\mathrm{dd}, 1 \mathrm{H}, J=8.0 \mathrm{~Hz}, J=9.5 \mathrm{~Hz}, \mathrm{H}-2^{\prime}\right), 5.27$ (d, 1H, $\left.J=10.7 \mathrm{Jz}, \mathrm{H}-1\right), 4.98(\mathrm{~d}, 1 \mathrm{H}, J=8.0$ Hz, H-1'), 4.83 (dd, 1H, $J=8.8 \mathrm{~Hz}, J=9.8 \mathrm{~Hz}, \mathrm{H}-3), 4.47$ (t, 1H, $J=10.0 \mathrm{~Hz}, \mathrm{H}-2), 4.38$ $(\mathrm{dd}, 1 \mathrm{H}, J=4.7 \mathrm{~Hz}, J=10.4 \mathrm{~Hz}), 4.01(\mathrm{t}, 1 \mathrm{H}, J=9.1 \mathrm{~Hz}, \mathrm{H}-4), 3.86$ (t, $1 \mathrm{H}, J=10.3 \mathrm{~Hz}$, H-6), 3.78 (d, 1H, J = 9.7 Hz, H-5), 3.73 (m, 1H, H-5), 3.49 (s, 3H, $\mathrm{CH}_{3} \mathrm{OMe}$ ), 2.64 (m, $\left.2 \mathrm{H}, \mathrm{CH}_{2} \mathrm{Set}\right), 1.14\left(\mathrm{t}, 3 \mathrm{H}, J=7.4 \mathrm{~Hz}, \mathrm{CH}_{3} \mathrm{SEt}\right) ;{ }^{13} \mathrm{C} \mathrm{NMR}\left(100 \mathrm{MHz}, \mathrm{CDCl}_{3}\right): \delta=166.9$, 165.4, 165.3, 164.2, 137.0, 133.7, 133.3, 133.2, 132.8, 131.2, 130.9, 129.6, 129.4, 128.7, 128.5, 1128.4, 128.3, 128.0, 126.0, 123.6, 122.7, 101.8, 100.0, 81.4, 81.3, 77.1, 72.3, 72.0, 71.9, 70.5, 70.0, 68.7, 53.7, 52.5, 23.5, 14.7. ES-MS: $m / z 966.3[\mathrm{M}+\mathrm{Na}]^{+}$.

Methyl (methyl 4- $O$-\{3-O-[methyl (2,3,4-tri- $O$-benzoyl- $\alpha$-D-glucopyranosyluronate)]4,6- $O$-benzylidene-2-deoxy-2-phtalimido- $\beta$-D-glucopyranosyl\}-2,3- $O$-benzyl- $\alpha$-Dglucopyranosyluronate) (31):

Trisaccharide $\mathbf{3 1}$ was synthesized from $\mathbf{2 9}$ and $\mathbf{3 0}$ as described for $\mathbf{2 6}$.

One-pot procedure: Methyl (2,3,4-tri- $O$-benzoyl- $\alpha$-D-glucopyranosyluronate) 27 (78 mg, $0.15 \mathrm{mmol}$ ), $\mathrm{Ph}_{2} \mathrm{SO}(67 \mathrm{mg}, 0.33 \mathrm{mmol})$, and TTBP $(37 \mathrm{mg}, 0.15 \mathrm{mmol})$ were dissolved in $\mathrm{DCM}(5 \mathrm{~mL})$ and the reaction mixture was cooled to $-60^{\circ} \mathrm{C} . \mathrm{Tf}_{2} \mathrm{O}(30 \mu \mathrm{L}, 0.16 \mathrm{mmol})$ was added and the reaction mixture was slowly brought to $-20^{\circ} \mathrm{C}$ in 1 hour, after which ethyl 4,6-O-benzylidene-2-deoxy-2-phtalimido-1-thio- $\beta$-D-glucopyranoside 28 (44 mg, $0.10 \mathrm{mmol}$ in $1 \mathrm{~mL} \mathrm{DCM}$ ) was added. The reaction mixture was slowly warmed to room temperature. After 1 hour at room temperature the reaction mixture was cooled to $-60^{\circ} \mathrm{C}$ and a solution of TTBP $(124 \mathrm{mg}, 0.50 \mathrm{mmol})$ and $\mathrm{Tf}_{2} \mathrm{O}(20 \mu \mathrm{L}, 0.12 \mathrm{mmol})$ were added. After the reaction was kept at $-60^{\circ} \mathrm{C}$ for 10 min, methyl (methyl 2,3-O-benzyl- $\alpha$-Dglucopyranosyluronate) $\mathbf{3 0}$ was added. The reaction was warmed to room temperature and stirred overnight, after which it was quenched with $\mathrm{Et}_{3} \mathrm{~N}(0.2 \mathrm{~mL})$. Standard work-up 
and purification gave the hyaluronan trisaccharide 31 (40 mg, $0.032 \mathrm{mmol}, 32 \%) . \mathrm{R}_{f} 0.50$ (50\% EtOAc in PE). $[\alpha]^{25}+4.6\left(c=0.70, \mathrm{CHCl}_{3}\right) ;{ }^{1} \mathrm{H} \mathrm{NMR}\left(400 \mathrm{MHz}, \mathrm{CDCl}_{3}\right): \delta=$ 7.94-7.18 (m, 34H, CHarom), 5.60 (t, 1H, $J=9.4$ Hz, H-3"), 5.41(t, 1H, $J=9.4 \mathrm{~Hz}, \mathrm{H}-4 ")$, $5.41(\mathrm{~s}, 1 \mathrm{H}, \mathrm{CH} \mathrm{Ph}), 5.27$ (dd, $1 \mathrm{H}, J=8.1 \mathrm{~Hz}, J=9.4 \mathrm{~Hz}, \mathrm{H}-2 "), 5.20$ (d, $1 \mathrm{H}, J=8.4 \mathrm{~Hz}$, H-1'), 4.93 (d, 1H, $J=8.8 \mathrm{~Hz}, \mathrm{CHH} \mathrm{Bn}), 4.91(\mathrm{~d}, 1 \mathrm{H}$, ), 5.27 (dd, $1 \mathrm{H}, J=8.1 \mathrm{~Hz}, J=9.4$ Hz, H-2"), 5.20 (d, 1H, J = 8.4 Hz, H-1'), 4.93 (d, 1H, J = 8.8 Hz, CHH Bn), 4.89 (d, 1H, $J=7.7 \mathrm{~Hz}, \mathrm{H}-1$ "), 4.77 (dd, 1H, $J=8.9 \mathrm{~Hz}, J=10.2 \mathrm{~Hz}, \mathrm{H}-3$ '), 4.72 (d, 1H, $J=12.2 \mathrm{~Hz}$, CHH Bn), 4.54 (d, 1H, $J=12.2 \mathrm{~Hz}, \mathrm{CH} H \mathrm{Bn}), 4.42$ (d, 1H, $J=3.5 \mathrm{~Hz}, \mathrm{H}-1), 4.28$ (dd, $\left.1 \mathrm{H}, J=8.4 \mathrm{~Hz}, J=10.2 \mathrm{~Hz}, \mathrm{H}-2^{\prime}\right), 4.02$ (m, 1H, H-6'), 3.88 (m, 2H, H-4, H-4'), 3.79 (m, 2H, H-3, H-5), 3.74 (d, 1H, J = 9.6 Hz, H-5"), 3.49 (s, 3H, $\mathrm{CH}_{3} \mathrm{OMe}$ ), 3.47 (m, 1H, H5), 3.43 (m, 2H, H-2, H-6'), 3.39 (s, $3 \mathrm{H}, \mathrm{CH}_{3} \mathrm{OMe}$ ), 3.25 (s, $3 \mathrm{H}, \mathrm{CH}_{3} \mathrm{OMe}$ ); ${ }^{13} \mathrm{C} \mathrm{NMR}$ $\left(100 \mathrm{MHz}, \mathrm{CDCl}_{3}\right): \delta=169.8,167.8,166.2,165.9,165.0,140.1,138.8,137.9,134.3$, 134.1, 134.0, 133.6, 132.2, 130.2, 129.6, 129.4, 129.3, 128.7, 128.1, 102.5, 100.7, 99.5, 99.0, 81.8, 80.2, 79.3, 78.8, 78.1, 76.9, 76.0, 74.5, 73.3, 72.8, 72.7,72.5, 70.8, 70.5, 69.4, 66.6, 56.5, 56.2, 53.4, 53.2; ES-MS: $\mathrm{m} / z 1306.5[\mathrm{M}+\mathrm{Na}]^{+}$.

(1) (1) Crich, D., Smith, M., Yao, Q., Picione, J. Synthesis 2001, 323-326.

(2) (a) Veeneman, G. H., Van Boom, J. H. Tetrahedron Lett. 1990, 31, 275-278. (b) Uchiro, H., Wakiyama, Y., Mukaiyama, T. Chem.Lett. 1998, 7, 567-568. 